\title{
Endoscope-assisted enucleation and guided bone regeneration of a recurrent aneurysmal bone cyst in the mandibular condyle
}

\author{
Xiao-Jie Diao ${ }^{1}$, Hua-Ming $\mathrm{Mai}^{2}$, Lei Zhang ${ }^{3}$, Xu-Dong Wang*3 \\ ${ }^{1}$ Department of Stomatology, Traditional Chinese Medical Hospital of Xinjiang Uygur Autonomous Region, Urumqi, Xinjiang, \\ People's Republic of China \\ ${ }^{2}$ Department of Oral and Maxillofacial Surgery, College of Stomatology, Guangxi medical University, Nanning, People's Republic \\ of China \\ ${ }^{3}$ Department of Oral \& Cranio-maxillofacial Science, Shanghai Ninth People's Hospital, Shanghai Jiao Tong University School of \\ Medicine, Shanghai Key Laboratory of Stomatology, Shanghai, People's Republic of China
}

Received: December 28, 2015

Accepted: March 27, 2016

Online Published: April 18, 2016

DOI: $10.5430 /$ css.v2n3p1

URL: http://dx.doi.org/10.5430/css.v2n3p1

\begin{abstract}
Aneurysmal bone cyst $(\mathrm{ABC})$ is a locally destructive, expansive, benign bone disease which most commonly occurs in the metaphyseal region of the long bones of children and adolescents, only $1 \%$ to $3 \%$ of ABCs have been described in the jaws. The aim of this study was to explore the minimal invasive surgical technique of treating $\mathrm{ABC}$ occurring in the mandibular condyle. Here we present a 10-year-old girl with recurrent $\mathrm{ABC}$ in her left condyle and adjacent ramus. She was treated by curettage via an extraoral endoscopic-assisted surgical approach and guided bone regeneration. The patient had an uneventful postoperative period with no injury to the facial nerves and minimal scarring. No recurrence was found in 4 years (from 2011). Computed tomography (CT) showed bone formation and remodeling of the condyle and ramus. Endoscopic-assisted curettage combined with guided bone regeneration can achieve an ideal effect in the treatment of $\mathrm{ABC}$ of the mandibular condyle and ramus.
\end{abstract}

Key Words: Aneurysmal bone cyst, Mandibular condyle, Endoscope

\section{INTRODUCTION}

Aneurysmal bone cyst (ABC) is a locally destructive, expansive, benign bone disease which most commonly occurs in the metaphyseal region of the long bones of children and adolescents. $\mathrm{ABC}$ constitutes approximately $1 \%$ of all bone tumors; only $1 \%$ to $3 \%$ of $\mathrm{ABCs}$ have been described in the jaws. ${ }^{[1]}$ The incidence of $\mathrm{ABC}$ is 0.14 per million. It is infrequently found in the mandibular condyle among all the cystic lesions.
The temporomandibular joint (TMJ) region contains a number of important and vulnerable structures including branches of the facial nerve. Compared with open surgery, endoscopicassisted technique recently was implemented in the TMJ region for fractures and other disorders has the benefit of less visible scarring, reduction in facial nerve deficit, and quicker functional rehabilitation. ${ }^{[2]}$ But the application of curettage of condylar cysts has been rarely reported. This clinical report describes a rare $\mathrm{ABC}$ involved in the mandibu-

\footnotetext{
*Correspondence: Xu-Dong Wang; Email: Xudongwang70@ @otmail.com; Address: Department of Oral \& Cranio-maxillofacial Science, Shanghai Ninth People's Hospital; Shanghai Jiao Tong University School of Medicine, Shanghai Key Laboratory of Stomatology, No.639 Zhizaoju Road, Shanghai 200011, People's Republic of China.
} 
lar condyle and ramus with the final treatment of curettage under endoscopic vision and introduction of bovine hydroxyapatite (Bio-Oss, Geistlich, Switzerland).

\section{CASE Representation}

\subsection{Patient}

A 10-year-old, otherwise healthy Chinese girl with no history of trauma or other TMJ problems presented to our hospital with a 2-month history of a painless swelling of the left TMJ region in February 2010. Preoperative Magnatic Resonance Imaging (MRI) showed a T1 hypointense and T2 hyperintense homogenous lesion with some internal septation in the left condyle and adjacent ramus (see Figure 1A). The lesion was measured $29 \mathrm{~mm} \times 22 \mathrm{~mm}$. A computed tomography (CT) scan showed thinned, but intact, cortical bone with multiple, thickened septations within the lesion (see Figures 1B and $1 \mathrm{C}$ ). Three-dimensional $\mathrm{CT}$ reconstruction revealed a multiloculated cystic mass in the condyle and upper half of the ramus (see Figure 1D). Radiologic differential diagnosis included cystic lesion and benign giant cell lesion. Frozensection examination revealed a hemorrhagic fibrovascular cyst wall with hemosiderin deposition and abundant multinucleated giant cells, features consistent with ABC. Therefore, curettage of the lesion was performed through the conventional submandibular incision. Eighteen months postsurgery, a follow-up CT scan revealed a destructive radiolucent lesion in the left condyle (see Figure 2A). The lesion was $28 \mathrm{~mm}$ $\times 15 \mathrm{~mm} \times 7 \mathrm{~mm}$ in size, larger than the $19 \mathrm{~mm}$-lesion scanned 6 months postoperatively. MRI (see Figure 2B) revealed two separate, T2 hyperintense homogenous masses in the condyle, without symptoms or patient discomfort. Diagnosed with a relapsed $\mathrm{ABC}$, the girl was subjected to a second surgery in August 2011.

\subsection{Surgical technique}

To decrease the possibility of aesthetic complication and circumvent facial nerve deficit, the operation was performed following the incision of a small part of the submandibular scar formed by the previous surgery. And considering of excessive bleeding, the operator would ligature the external carotid artery from the previous scar if that occur. Through endoscopic viewing using $30^{\circ}$ angled optics, we were able to visualize the lesion at the level of the condyle (see Figure $3 \mathrm{~A}$ and $3 \mathrm{~B}$ ), the lesion was completely curetted with the use of curved tools. Subsequent to providing hemostasis and irrigation, we filled the residual cavity with lyophilised bovine heterologous bone particles (Bio-Oss), followed by covering with resorbable collagen membrane (Bio-Gide, Geistlich, Switzerland) to induce osteogenesis (see Figure 3C and 3D). Primary closure of the surgical wound was done in layers after scar revision and insertion of a single, negative-pressure drainage tube. Prophylactic antibiotics were administered, as well as corticoids, on the following days.

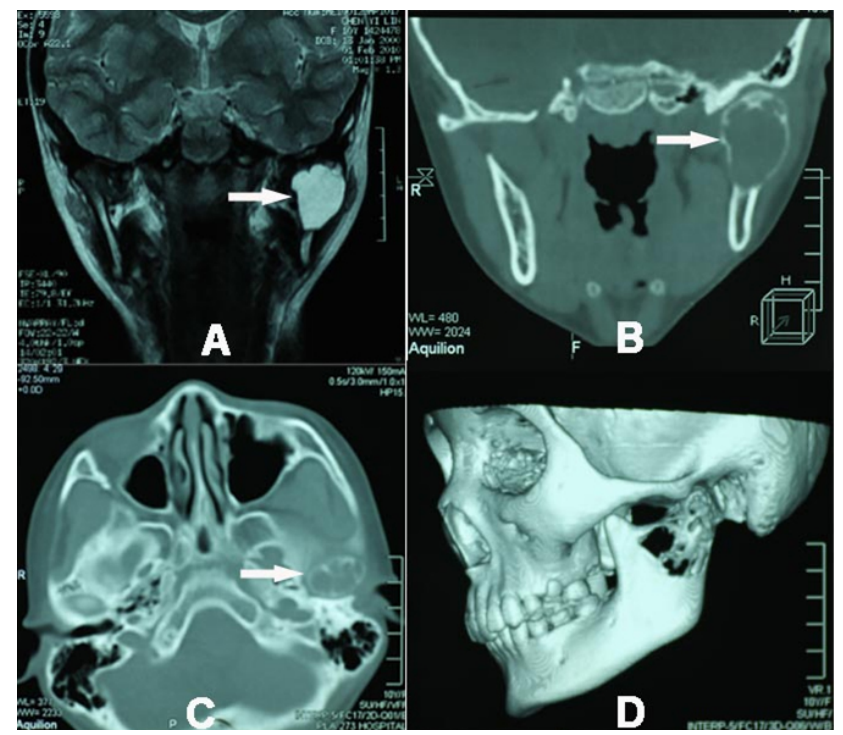

Figure 1. Preoperative imaging, with a white arrow indicating the lesion

(A) T2-weighted MRI shows left condyle widely filled with a hyperintensive signals, indicative of liquid;

(B) CT scan shows that the cortical bone was thinned but intact; (C) There were multiple, thickened septations within the lesion; (D) 3-dimensional CT reconstruction revealed a multiloculated, cystic mass in the condyle and upper half of the ramus

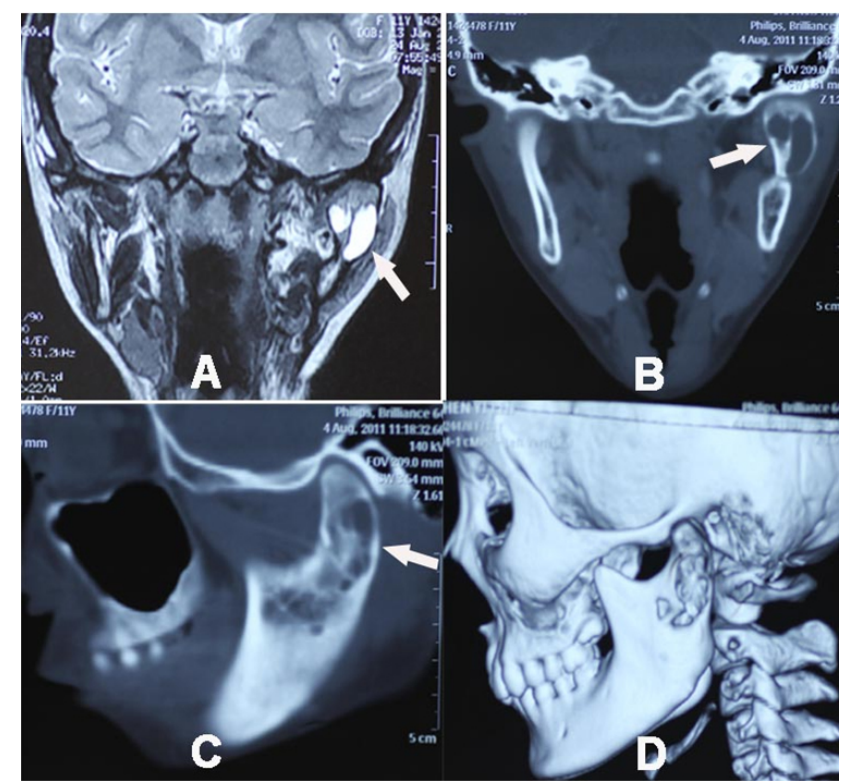

Figure 2. Imaging at 18 months after first surgery (A) MRI revealed two separate T2 hyperintense homogenous masses in the condyle; $(B-D) C T$ scan revealed destructive radiolucent lesion in the left condyle 


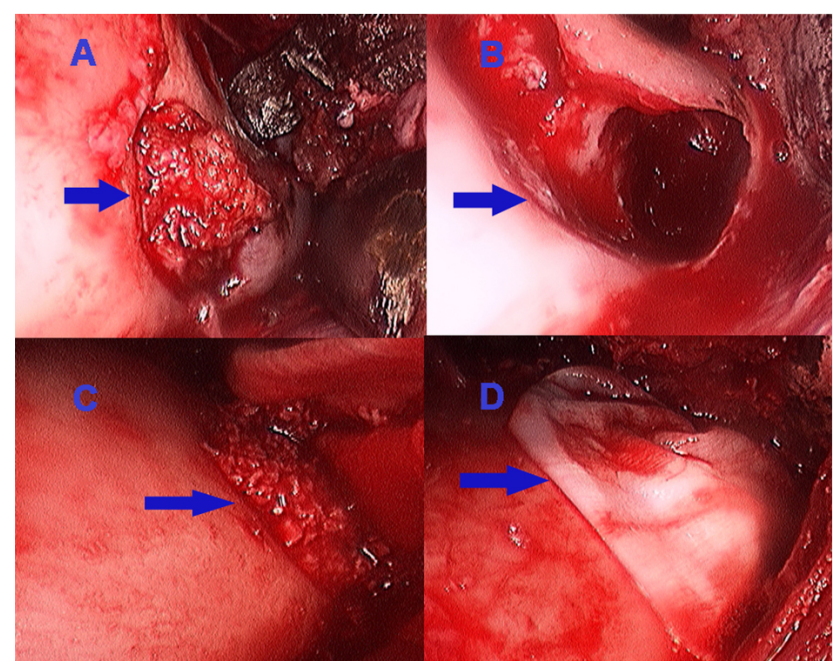

Figure 3. Stages of endoscope-assisted surgery with a blue arrow indicating the lesion

(A) Visualisation of the lesion in the condyle; Residual cavity (B) was filled with Bio-Oss $(C)$, followed by covering with Bio-Gide collagen membrane $(D)$

\section{Results}

The patient had an uneventful postoperative period without injury to her facial nerves and minimal scarring. Her drain was removed on the second postoperative day. She was well enough to be discharged 4 days postprocedure. Biopsy revealed muscle tissue and tumorlike hyperplasia. No recurrence was found in 4 years (from 2011). CT showed bone formation and remodeling of the condyle and ramus (see Figure 4).

\section{Discussion}

$\mathrm{ABC}$ is a rare, rapidly growing and destructive benign bone tumor that occurs most frequently in patients under 20 years of age. In the Cottalorda et al. demographic data, $62.7 \%$ of $\mathrm{ABCs}$ occurred in the long bones: the femur, tibia, spine, humerus, pelvis, and fibula were the most common location. ${ }^{[3]}$ Only $2 \%$ of the lesions appear in the jaws ${ }^{[4]}$ and the presence of $\mathrm{ABC}$ in the mandibular condyle is quite rare. Histologically, ABC is classified as a pseudocyst composed of blood-filled chambers surrounded and traversed by benign-appearing macrophages, lymphocytes, fibroblasts, bone-forming cells, and osteoclastlike multinucleated giant cells without epithelial lining. ${ }^{[5,6]}$

MRI and CT are helpful in radiographic differential diagnosis because MRI in typical cases shows double-density fluid levels within the cyst and internal septation of the cyst with soap-bubble features (particularly in T2-weighted images), ${ }^{[7]}$ whereas the expansive character of the lesion and its thin bony walls are better depicted by CT.

Even if plain radiographs and MRI support the diagnosis of ABC, biopsy is imperative because other tumors, such as telangiectatic osteogenic sarcoma, may be misdiagnosed as ABCs.

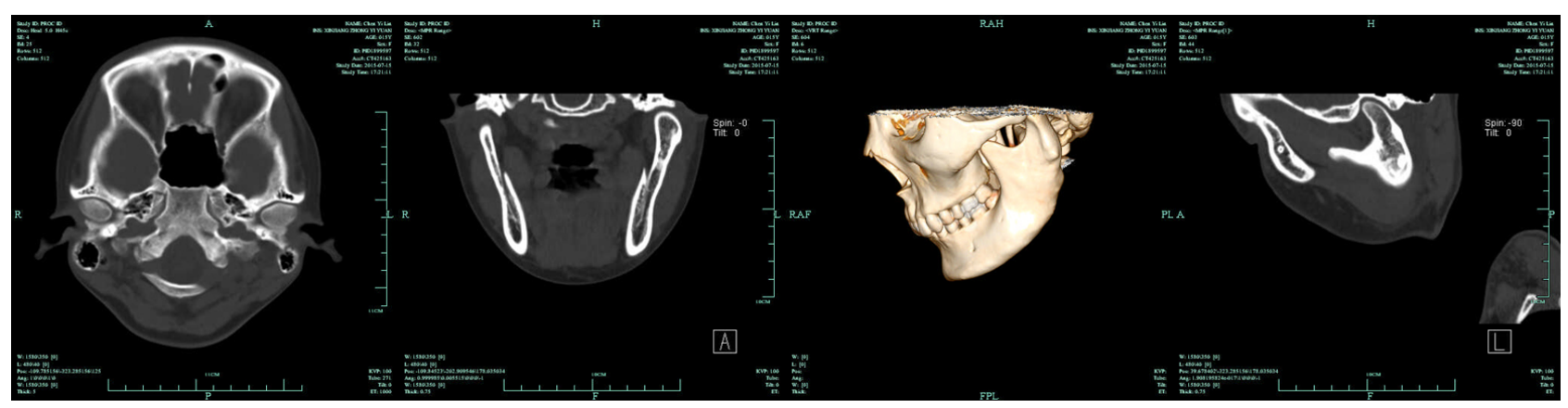

Figure 4. Postoperative imaging 4 years later CT shows bone formation in the condyle and ramus

There are various alternative forms of treatment which deserve discussion including intralesional curettage, subtotal or total resection, sclerotherapy and selective/superselective embolisation. Percutaneous sclerotherapy using agents such as Ethibloc (a hydroalcoholic radiopaque solution of zein no longer available), policodanol, and absolute alcohol have been described as highly effective and acceptable alternatives, having the advantages of less invasiveness, less pain, shorter hospital stay, lower healing time, fewer complications and a better functional outcome. But the potential complications include necrosis at the injection site in case of extravasation, pulmonary embolism, osteomyelitis, and, especially, adjacent nerve palsy in the TMJ region through which branche of the facial nerve run.

Standard treatment of accessible extremity aneurysmal bone cysts is intralesional curettage. Pawel Flont et al. compared cyst wall curettage and en bloc excision in pain, limb length differences, reduced range of motion and muscle strength 
impairment. The result is that the differences were not statistically significant $(p>.05) .{ }^{[8]}$ Curettage with or without bone grafting is the mainstay of treatment. ${ }^{[9]}$ Recurrence rates after curettage have varied widely in different reports. According to a review of $690 \mathrm{ABCs}$ treated with curettage and bone grafting and summarised by Cottalorda et al.,${ }^{[10]}$ the recurrence rate in this series varied from $18 \%$ to $59 \%$; the overall recurrence rate was $31 \%$. However, in a retrospective clinical study of 17 cases of ABCs of the mandible treated with complete surgical curettage without bone grafting, there were no recurrences. The recurrence rate may be related to the location of the lesions, diameter of the cortical window, and individual operative procedures. Before entering the lesion, one should adequately expose it to enable a rapid curettage. The large diameter of the cortical window may facilitate observation of the interior of the cyst and result in a low recurrence rate. ${ }^{[11]}$ En bloc resection is the treatment having the lowest risk of local recurrence, but it must be considered only when there is no other treatment option because of the loss of the condyle and subsequent growth arrest of the mandible in such case.

With the advantages of minimally invasive surgery - decreased morbidity, improved visualisation, surgical precision, reduction in facial nerve deficit, and quicker functional rehabilitation - endoscopic surgery has been developed in the specialty of maxillofacial surgery mostly for trauma, orthognathic surgery, sialoendoscopy, and TMJ disease. However, only two papers describe the removal of mandibular or condylar lesions; the present case is the first case describing the application of curettage of ABCs. Because of the need to trim the scar left by the first surgery, extroral access was designed to insert the endoscope through a small submandibular scar incision and smoothly reach the operating field, providing a detailed magnification of the images. Thus, complete curettage of the lesion was attained with reliable instrumental support.

Given that there was a deep cavity left after curettage of the cyst and there had been recurrence, the cavity was filled with Bio-Oss (a natural bone substitute obtained from the mineral portion of bovine bone that closely resembles human bone), resulting in effective bone regeneration. This idea was encouraged by the reports of Delloye and coworkers, ${ }^{[12,13]}$ who described 13 cases of ABCs receiving grafts of demineralised bone and autogenous bone marrow to induce healing of the cyst without curettage. Eleven cases had successful healing after a mean follow-up period of 3.9 years. The rationale underlying this intralesional treatment was the expectation that the bone-grafting material would reverse cystic expansion by promoting ossification through a bone-induction mechanism. To our knowledge, this is the first case describing the clinical application of guided bone regeneration of a cyst of the mandiblular condyle.

\section{Conclusions}

Although this procedure needs more long-term follow-up confirmation, endoscopic-assisted curettage combined with grafting of bovine heterologous bone particles may become the ideal treatment for the $\mathrm{ABC}$ of the mandibular condyle and ramus.

\section{REFERENCES}

[1] Jundt G, Prein J. Bone tumors and tumor-like lesions of the jaw Findings from the Basel DOSAK reference registry. Mund Kiefer Gesichtschir 2000; 4 Suppl 1: S196-207. PMid: 10938660. http: //dx.doi.org/10.1007/PL00014541

[2] Schmelzeisen R, Cienfuegos-Monroy R, Schon R, et al. Patient benefit from endoscopically assisted fixation of condylar neck fractures-a randomized controlled trial. J Oral Maxillofac Surg. 2009; 67: 147 158. PMid: 19070761. http://dx.doi.org/10.1016/j.joms . 2008.09.019

[3] Cottalorda J, Kohler R, Sales de Gauzy J, et al. Epidemiology of aneurysmal bone cyst in children: a multicenter study and literature review. J Pediatr Orthop B. 2004; 13: 389-94. PMid: 15599231. http://dx.doi.org/10.1097/01202412-200411000-00008

[4] Kaffe I, Naor H, Calderon S, et al. Radiological and clinical features of aneurysmal bone cyst of the jaws. Dentomaxillofac Radiol. 1999; 28: 167-172. PMid: 10740472. http://dx.doi.org/10.1038/s j.dmfr. 4600434

[5] Mendenhall WM, Zlotecki RA, Gibbs CP, et al. Aneurysmal bone cyst. Am J Clin Oncol. 2006; 29: 311-15. PMid: 16755186. http: //dx.doi.org/10.1097/01.coc.0000204403.13451.52

[6] Mankin HJ, Hornicek FJ, Ortiz-Cruz E, et al. Aneurysmal bone cyst: a review of 150 patients. J Clin Oncol. 2005; 23: 6756-6762. PMid: 16170183. http://dx.doi.org/10.1200/JC0.2005.15.255

[7] Sullivan RJ, Meyer JS, Dormans JP, et al. Diagnosing aneurysmal and unicameral bone cysts with magnetic resonance imaging. Clin Orthop Relat Res. 1999: 186-190. PMid: 10627734 http://dx.doi.org/10.1097/00003086-199909000-00024

[8] Flont P, Kolacinska-Flont M, Niedzielski K. A comparison of cyst wall curettage and en bloc excision in the treatment of aneurysmal bone cysts. World Journal of Surgical Oncology. 2013; 11(10): 238242. PMid: 23701661. http://dx.doi.org/10.1186/1477-7 819-11-109

[9] Dios AMVD, Bond JR, Shives TC, et al. Aneurysmal bone cyst. A clinicopathologic study of 238 cases. Cancer. 1992; 69(12): 29212931. http://dx.doi.org/10.1002/1097-0142(19920615)6 $9: 12<2921::$ AID-CNCR2820691210>3.0.C0;2-E

[10] Cottalorda J, Bourelle S. Current treatments of primary aneurysmal bone cysts. J Pediatr Orthop B. 2006; 15: 155-167. 
PMid: 16601582. http://dx.doi.org/10.1097/01.bpb.0000 210588.50899.29

[11] Dormans JP, Hanna BG, Johnston DR. Surgical treatment and recurrence rate of aneurysmal bone cysts in children. Clin Orthop Relat Res. 2004: 205-211. PMid: 15123949. http://dx.doi.org/10. 1097/01.blo.0000126336.46604.e1

[12] Delloye C, De Nayer P, Malghem J, et al. Induced healing of aneurys- mal bone cysts by demineralized bone particles. A report of two cases Arch Orthop Trauma Surg. 1996; 115: 141-145. PMid: 8861578 http://dx.doi.org/10.1007/BF00434541

[13] Docquier PL, Delloye C. Treatment of aneurysmal bone cysts by introduction of demineralized bone and autogenous bone marrow. J Bone Joint Surg Am. 2005; 87: 2253-2258. PMid: 16203891 http://dx.doi.org/10.2106/JBJS.D.02540 